
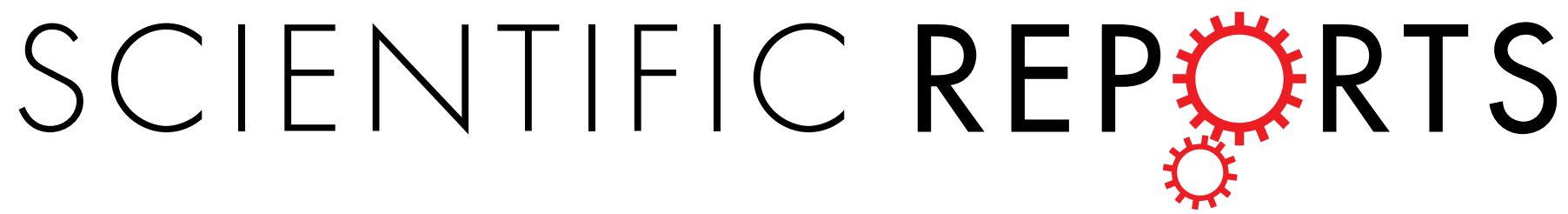

eEF1A1 binds and enriches protoporphyrin IX in cancer cells in 5 -aminolevulinic acid based

Received: 04 February 2016

Accepted: 08 April 2016

Published: 06 May 2016

\section{photodynamic therapy}

\author{
Zhichao Fan ${ }^{1,2,7, *}$, Xiaojun Cui $i^{2,3, *}$, Dan Wei ${ }^{1}$, Wei Liu ${ }^{6}$, Buhong $\mathrm{Li}^{4}$, Hao He ${ }^{1}$, Huamao Ye ${ }^{5}$,
} Naishuo $\mathrm{Zhu}^{2,3}$ \& Xunbin Wei, ${ }^{1,2}$

Photodynamic therapy (PDT) with protoporphyrin IX (PpIX), which is endogenously derived from 5-aminolevulinic acid (5-ALA) or its derivatives, is a promising modality for the treatment of both pre-malignant and malignant lesions. However, the mechanisms of how ALA-induced PpIX selectively accumulated in the tumors are not fully elucidated. Here we discovered that eukaryotic translation elongation factor 1 alpha 1 (eEF1A1) interacted with PpIX (with an affinity constant of $2.96 \times 10^{6} \mathrm{M}^{-1}$ ). Microscopy imaging showed that ALA-induced PpIX was co-localized with eEF1A1 in cancer cells. eEF1A1 was found to enrich ALA-induced PpIX in cells by competitively blocking the downstream bioavailability of PpIX. Taken together, our study discovered eEF1A1 as a novel photosensitizer binding protein, which may play an essential role in the enrichment of ALA-induced PpIX in cancer cells during PDT. These suggested eEF1A1 as a molecular marker to predict the selectivity and efficiency of 5-ALA based PDT in cancer therapy.

Photodynamic therapy (PDT) is increasingly being recognized as an attractive and alternative treatment modality for cancers ${ }^{1-5}$. 5-aminolevulinic acid (5-ALA), as the precursor of protoporphyrin IX (PpIX) in heme biosynthesis pathway ${ }^{6}$, is one of the most important reagents used in cancer PDT of the prostate ${ }^{7}$, breast ${ }^{8}$, $\operatorname{skin}^{9,10}$, cervix ${ }^{11}$, bladder $^{12}$, brain ${ }^{13,14}$, lung ${ }^{15}$, stomach ${ }^{16}$, head and neck ${ }^{17}$, and colon ${ }^{18}$, as well as those for leukemia ${ }^{19}$ and other types of cancer.

Many studies have aimed on the mechanism of 5-ALA-induced PpIX selective accumulation in tumor lesion. One hypothesis focuses on the target proteins which can interact with PpIX: the peripheral benzodiazepine receptor $(\mathrm{PBR})^{19}, \mathrm{p} 53^{18}$, fragile histidine triad protein $(\mathrm{FHIT})^{20}$ or low density lipoprotein (LDL) has been proposed as target proteins. Some of these proteins, such as LDL, can be enriched from blood into tumor cells compared to normal tissues ${ }^{21}$. Other studies have shown positive or negative correlations between PpIX accumulation and peptide transporter 1 (PEPT1) $)^{12}$ or ATP-binding cassette sub-family G member 2 (ABCG2) ${ }^{12,13}$ expressions, respectively. However, some of the studies above are inconsistent. For example, p53 and FHIT are demonstrated as tumor suppressors, which are rarely expressed in cancer cells. Therefore, the mechanism of how 5-ALA-induced PpIX accumulated selectively in tumor lesions is unclear and remain further investigated.

Using the method of complementary DNA (cDNA) phage displayed library screening ${ }^{22-25}$, we discovered a novel PpIX binding protein - eukaryotic translation elongation factor 1 alpha 1 (eEF1A1), which is highly expressed in cancer cells ${ }^{26}$. Moreover, we measured the affinity and studied subcellular localization of eEF1A1 and

\footnotetext{
${ }^{1}$ State Key Laboratory of Oncogenes and Related Genes, Shanghai Cancer Institute, School of Biomedical Engineering, Shanghai Jiao Tong University, Shanghai, China. ${ }^{2}$ Institutes of Biomedical Sciences, Fudan University, Shanghai, China. ${ }^{3}$ Laboratory of Molecular Immunology, State Key Laboratory of Genetic Engineering, School of Life Sciences, Fudan University, Shanghai, China. ${ }^{4}$ Key Laboratory of Optoelectronic Science and Technology for Medicine of Ministry of Education, Fujian Provincial Key Laboratory for Photonics Technology, Fujian Normal University, Fuzhou, Fujian, China. ${ }^{5}$ Department of Urology, Changhai Hospital, Second Military University, Shanghai, China. ${ }^{6}$ Cell Death and Survival Networks Program, Sanford Burnham Prebys Medical Discovery Institute, La Jolla, California, USA. ${ }^{7}$ Division of Inflammation Biology, La Jolla Institute for Allergy \& Immunology, La Jolla, California, USA. *These authors contributed equally to this work. Correspondence and requests for materials should be addressed to N.Z. (email: nzhu@fudan.edu.cn) or X.W. (email: xwei01@sjtu.edu.cn)
} 
a

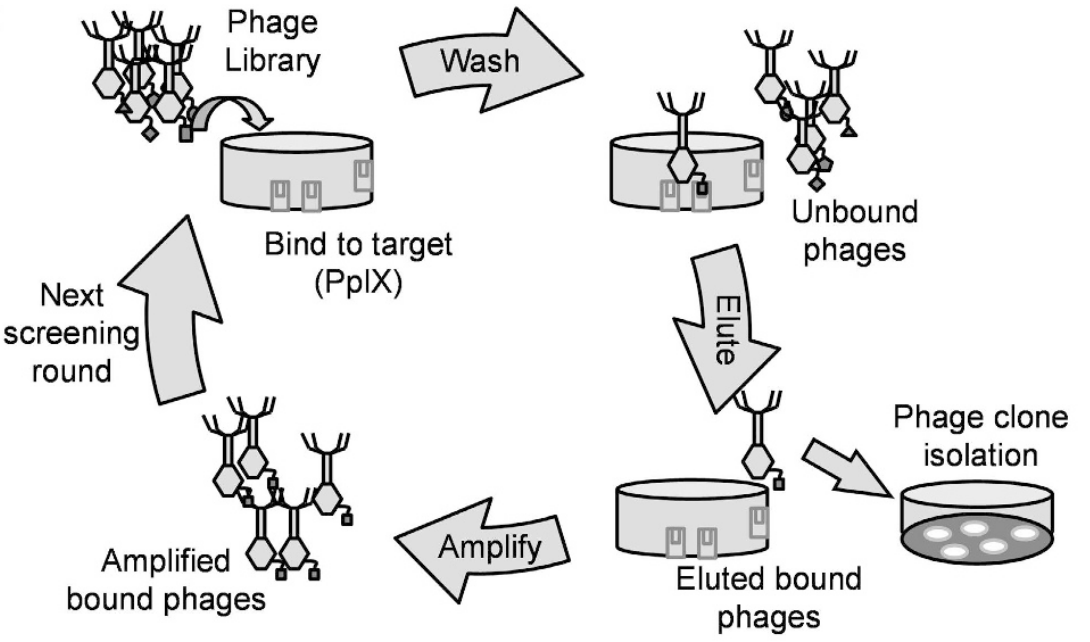

b

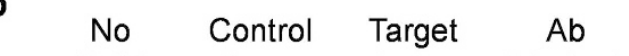

C

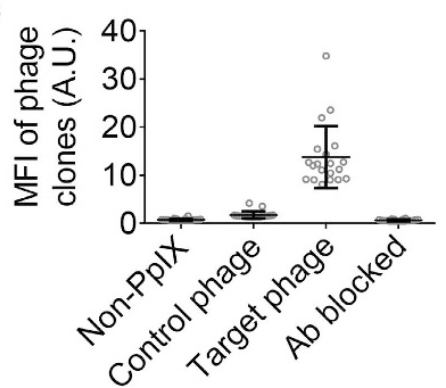

Figure 1. Screening of PpIX affinitive protein by T7Select Human Liver cDNA Library. (a) The cartoon diagrammatic drawing shows the processes of screening. (b) Typical fluorescence microscopy images verify the PpIX binding specificity of the protein expressed on selected phage clone. Selected phage clone shows significant PpIX binding (red) compared to no-PpIX-added and non-specific phage clones. eEF1A1 antibody blockade eliminate the PpIX binding. Scale bar is $10 \mu \mathrm{m}$. (c) The PpIX MFI of $n=20$ phage clones in each data set. Mean \pm SD.

PpIX. Overexpression of eEF1A1 was used to assay its function in the accumulations of 5-ALA-induced PpIX in cancer cells. In addition, we studied the mechanism of tumor-selective PpIX accumulation after exogenous intake of 5-ALA.

\section{Results}

eEF1A1 was identified as the novel target binding protein for PpIX. To investigate the mechanism of PpIX accumulation in PDT targeted cells, we sought to find out the PpIX-binding protein(s). Using phage displayed cDNA library technology ${ }^{22-25}$, we obtained the phage clone with the strongest and most selective PpIXbinding ability after four rounds of screening (Fig. 1a). Fluorescence microscopy imaging showed (Fig. 1b,c) that our selected clone has significant PpIX binding, which had considerable PpIX florescence intensity (mean florescence intensity, MFI $=13.75 \pm 6.43$ arbitrary units, A.U., $n=20$ ), compared to the clone of PpIX non-specific phages (MFI $=1.75 \pm 0.75$ A.U., $\mathrm{n}=20$ ).

To identify the peptide displayed on selected phage surface, the cDNA inserted into the phages were amplified by polymerase chain reaction (PCR) and sent for sequencing. After sequence alignment in GeneBank by basic local alignment search tool (BLAST), we found out that the peptide expressed on the selected phage (IKAVDKKAAGAGKVTKSAQKAQKAK) was the C terminal lysine motif of eEF1A1 and rich in basic amino acids. Blocking the peptides by an anti-eEF1A1 C terminal polyclonal antibody eliminated the PpIX-binding to the selected phage clones (Fig. 1b,c, MFI $=0.62 \pm 0.18$ A.U., $n=20$ ). Therefore, we have identified eEF1A1 as a PpIX-binding protein.

eEF1A1 interacts with ALA-induced PpIX. To evaluate the binding between eEF1A1 and PpIX, we first measured the fluorescence of PpIX bond to immobilized eEF1A1 protein. Compared to bovine serum albumin (BSA) control, eEF1A1 presented significant higher binding of PpIX (Fig. 2a), which was quantified by MFI. A linear correlation was observed between MFI of bound PpIX and amount of coated eEF1A1 $\left(\mathrm{R}^{2}=0.9736\right)$. In BSA control, the increase of bond PpIX was much less noticeable, which represented the non-specific binding of PpIX. A linear correlation in control group was also observed $\left(\mathrm{R}^{2}=0.9571\right)$, whereas $\sim 11.7$ times lower slope was shown compared to eEF1A1 group. Significant differences were present when comparing the regression lines of two groups $(\mathrm{P}<0.0001)$ using F-test and comparing the data sets of two groups in each concentration points (400-100 ng/100 $\mathrm{ll}, \mathrm{P}<0.01)$ using t-test. 
a

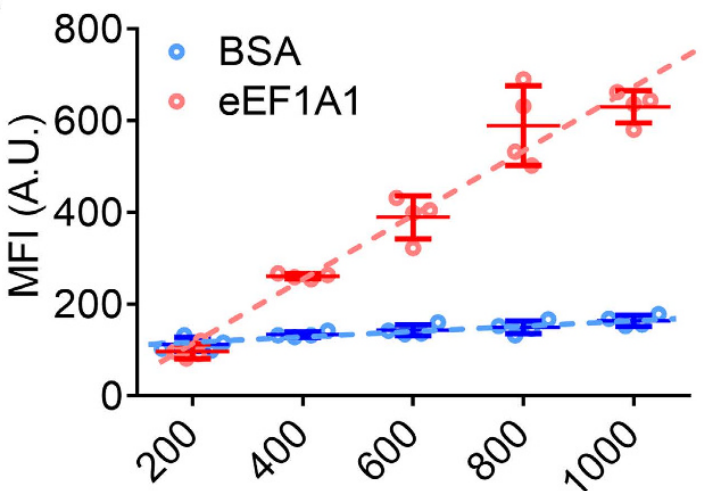

Protein coating concentration (ng/100 $\mu \mathrm{L})$

b

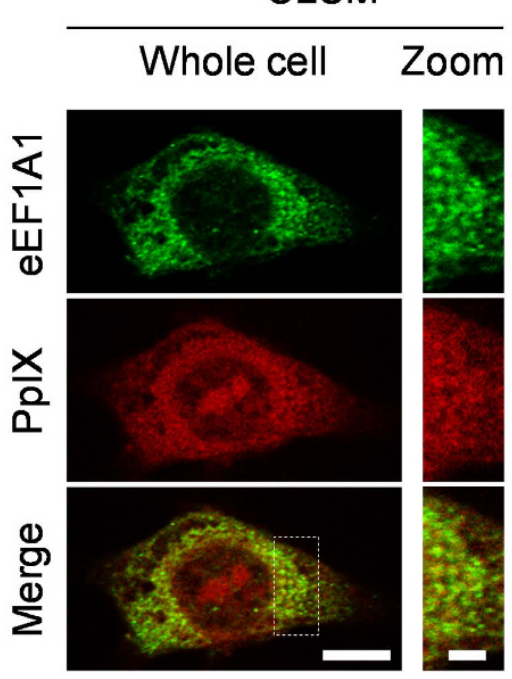

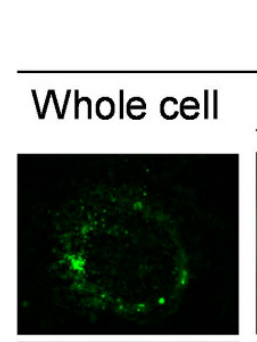
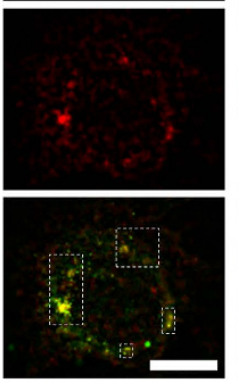

SIM

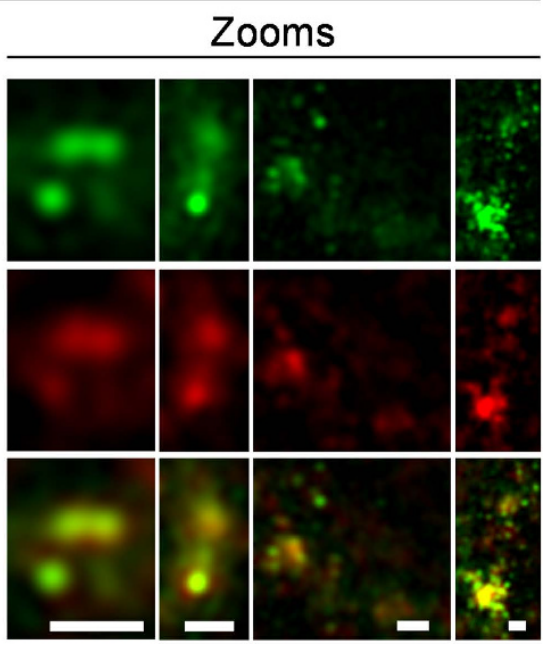

Figure 2. In vitro analysis of the interaction between eEF1A1 and PpIX. (a) Correlations of coated purified eEF1A1 protein and BSA (control) or binding PpIX (fluorescence intensity). $\mathrm{n}=4$ samples per data set; Mean \pm SD. (b) The representative images present the co-localization of FITC-labeled eEF1A1 (green) and PpIX (red) in situ acquired by both CLSM and SIM. Areas highlighted by white dot frames in whole cell images are zoomed to show details. Scale bars for whole cell images are $10 \mu \mathrm{m}$. Scale bar for zoomed CLSM image is $3 \mu \mathrm{m}$. Scale bars for zoomed SIM images are $1 \mu \mathrm{m}$.

Surface plasmon resonance (SPR) assay was performed to quantify the affinity constant (association constant, $\mathrm{K}_{\mathrm{a}}$ ) of eEF1A1-PpIX binding. A $\mathrm{K}_{\mathrm{a}}$ of $2.96 \times 10^{6} \mathrm{M}^{-1}$ was obtained in eEF1A1-PpIXbinding, which was significantly higher than the $\mathrm{K}_{\mathrm{a}}$ of PpIX-BSA $\left(\sim 1.8-2.3 \times 10^{5} \mathrm{M}^{-1} \text {, ref. } 37\right)^{27}$, PpIX-HSA (Human Serum Albumin, $\left.\sim 1.5-2.1 \times 10^{5} \mathrm{M}^{-1}\right)^{27}$, or PpIX-IgG (immunoglobulin G, 1.9-2.4 $\times 10^{5} \mathrm{M}^{-1}$ ) ${ }^{27}$, and even higher than some antibody-antigen binding ${ }^{28}$.

Immunofluorescence staining of 5-ALA incubated cells was utilized to investigate whether this eEF1A1-PpIX interaction was present in cells. Using confocal laser scanning microscopy (CLSM) and structured illumination microscopy (SIM) ${ }^{29}$, co-localizations of PpIX and eEF1A1 were observed in HepG2 cells after 24-hour incubation with 5-ALA (Fig. 2b). Most PpIX was co-localized with eEF1A1 protein in the cytoplasm, except for a small portion of PpIX located in the nucleus.

To further validate the co-localization of PpIX and eEF1A, co-dependence was obtained by evaluating Pearson's correlation coefficient ${ }^{30}$ for PpXI and eEF1A1 staining. A coefficient of $0.58 \pm 0.09(n=5)$ indicated a significant correlation between PpXI and FITC-labeled anti-eEF1A1-antibody pixel intensity profiles. This was further supported by larger Manders' overlap coefficients of $0.84 \pm 0.03(\mathrm{n}=5)$ and co-localization coefficient of $0.92 \pm 0.09$ and $0.96 \pm 0.07(\mathrm{n}=5)$ for M1 (indicating the co-localization of PpXI with anti-eEF1A1-FITC) and M2 (indicating the co-localization of anti-eEF1A1-FITC with PpXI), respectively ${ }^{30}$. The above analytical procedures avoid cursory assignment of PpXI and eEF1A1 co-localization merely by inspection.

We further performed intensity correlation analysis (ICA) ${ }^{31}$, which is another important objective measure of co-dependent staining between a pair of fluorescence channels and involves computation of intensity changes of 


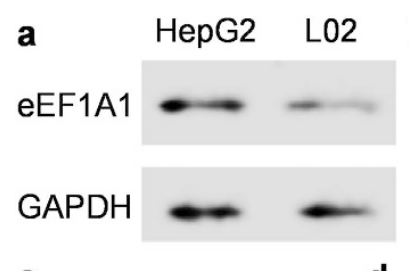

b eEF1A1 Control

C

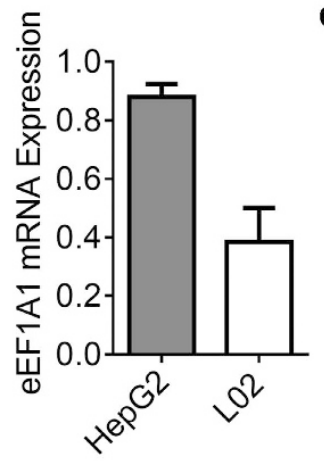

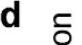
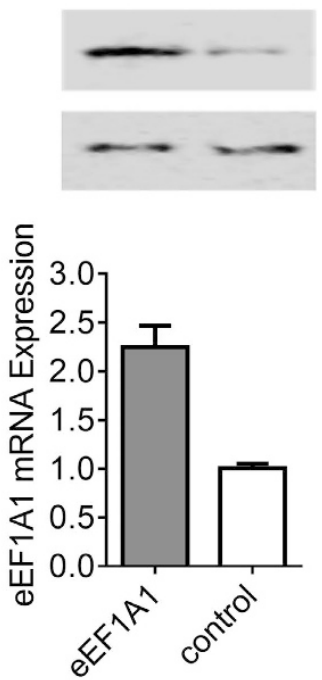

e

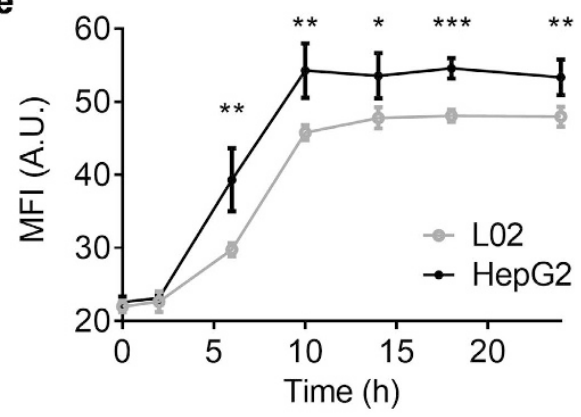

f

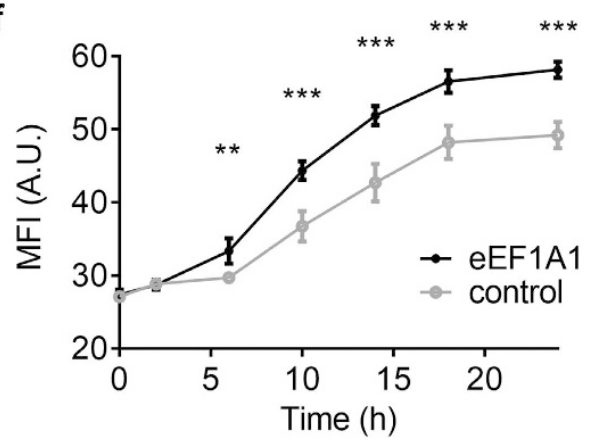

Figure 3. PpIX accumulation in living cells correlated to the expression of eEF1A1. (a-d) The expression of eEF1A1 in HepG2 and L02 cells (a,c) or HepG2 cells transfected by eEF1A1 reconstructed or control plasmids $(\mathbf{b}, \mathbf{d})$ assessed by western blot $(\mathbf{a}, \mathbf{b})$ and qRT-PCR $(\mathbf{c}, \mathbf{d})$. (e,f) Time-lapse measurements of ALA-induced PpIX accumulations in HepG2 and L02 cells (e) or HepG2 cells transfected by eEF1A1 reconstructed or control plasmids (f). Mean $\pm \mathrm{SD}, \mathrm{n}=4$ samples per data set, ${ }^{*} \mathrm{p}<0.05,{ }^{\star *} \mathrm{p}<0.01,{ }^{* *} \mathrm{p}<0.001$

corresponding pixels in each channel. The resulting intensity correlation quotient (ICQ) was $0.22 \pm 0.02(n=5)$, which indicated that PpXI and eEF1A1 were co-localized and varied synchronically.

eEF1A1 promotes the accumulation of ALA-induced PpIX in cells. To investigate the function of the strong interaction between eEF1A1 and PpIX, we studied the relationship between eEF1A1 expression and accumulation of the ALA-induced PpIX in cells. Hepatocellular carcinoma cells are known to express higher eEF1A1 than normal hepatocytes ${ }^{32}$. Indeed, more eEF1A1 were expressed in HepG2 cancer cell line than L02 noncancerous hepatic cell line, which was assessed by both quantitative reverse transcription-polymerase chain reaction (qRT-PCR, Fig. 3a) and western blotting (Fig. 3c). Correspondently, by measuring PpIX fluorescent intensity, we found out that more ALA-induced PpIX accumulated in HepG2 cells than L02 cells (Fig. 3e). Specifically, the ALA-induced PpIX increased dramatically after the addition of 5-ALA in HepG2 cells and reached the saturation at about hour 10. In contrast, the ALA-induced PpIX in L02 cells increased slower than that in HepG2 cells and reached to the peak at about hour 14. Significant differences between the two groups started to manifest from hour six. 


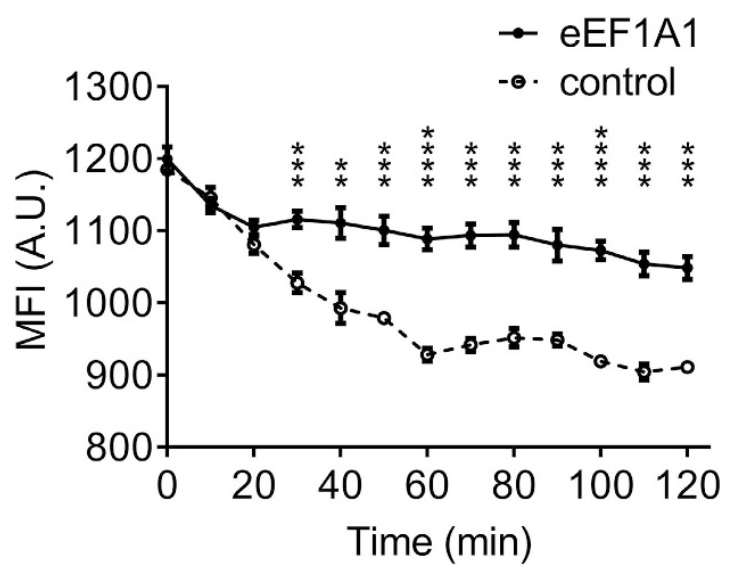

Figure 4. PpIX degradation in cell lysates was inhibited by eEF1A1. Time-lapse monitoring of PpIX MFI in lysates with or without adding eEF1A1 protein. PpIX is degraded significantly slower when eEF1A1 is presentcompared to that in the control. Mean $\pm \mathrm{SD}, \mathrm{n}=3$ samples per data set, ${ }^{* *} \mathrm{p}<0.01,{ }^{* * *} \mathrm{p}<0.001$, ${ }_{* * * *} \mathrm{p}<0.0001$.

To demonstrate whether this increasing enrichment was related to eEF1A1, we overly expressed eEF1A1 protein in HepG2 cells. Compared to HepG2 transfected with empty plasmid, HepG2 transfected with eEF1A1-reconstituted plasmid produced higher eEF1A1 messenger RNA (mRNA, Fig. 3b) and expressed higher amount of eEF1A1 protein (Fig. 3d). Interestingly, eEF1A1 over-expressed HepG2 showed higher amount of ALA-induced PpIX accumulation than control HepG2 (Fig. 3f). Significant differences between the two groups were observed from hour 5.

eEF1A1 prevents PpIX degradation. We demonstrated that eEF1A1 interacted with ALA-induced PpIX and promoted accumulation of ALA-induced PpIX in cells. We hypothesized that binding to eEF1A1 would prevent PpIX bioavailability in the downstream metabolism. By measuring PpIX degradation in HepG2 cell lysate, we found out that adding purified eEF1A1 protein significantly inhibited PpIX degradation compared to vehicle control (Fig. 4). Significant differences between the two groups were observed from minute 30 .

\section{Discussion}

Our study has identified eEF1A1 as a novel binding protein for photosensitizer ALA-induced PpIX. eEF1A1 was further confirmed to have a very strong binding affinity to PpIX, which is close to or exceeds the affinity between antigen and antibody $\left(10^{5} \sim 10^{7} \mathrm{M}^{-1}\right)^{28}$. Moreover, our study has demonstrated that the eEF1A1-PpIX interactions were present in cancer cells and led to the accumulation of ALA-induced PpIX by preventing PpIX degradation. Since the tumorogenic potential of eEF1A1 has been proposed following the observation that its over-expression correlates with increased metastatic potential in mammary adenocarcinoma ${ }^{26,32,33}$, including melanomas, hepatocellular carcinoma, pancreatic carcinoma, breast carcinoma, prostatic carcinoma, cervical carcinoma and bladder carcinoma, some of which were commonly treated by PDT clinically, our finding provide an mechanistic explanation that why PpIX accumulated in cancer cells in 5-ALA based PDT.

On the other hand, eEF1A1 might serve as an target for 5-ALA based PDT. The eEF1A, including eEF1A1 and eEF1A2, are the second most abundant protein (1-3\% of total protein content) after actin and an important component of translation machinery ${ }^{34}$. Canonical function of eEF1A1 serves as a translation factor, which delivers the aminoacylated- transfer-RNA to its binding site of the ribosome for decoding of mRNA by codon-anticodon interactions ${ }^{34}$ and plays key roles in protein synthesis ${ }^{26}$. Its protein sequence is highly conserved among eukaryotic species ranging from yeast to human. In addition to its critical role in protein biosynthesis, eEF1A1 has been implicated in broadly uncanonical function, including actin-binding and bundling ${ }^{35}$, apoptosis ${ }^{36}$, nuclear transport ${ }^{37}$, heat shock response ${ }^{37}$, cell anoikis ${ }^{38}$ and proteasomal-mediated degradation of damaged proteins $^{39}$. eEF1A1 is required for normal cell growth, proliferation, and cell cycle progression ${ }^{40}$. Indeed, eEF1A1 was reported as a target for anti-tumor therapy ${ }^{41}$. Targeting eEF1A1 can combat apoptosis-resistant cancers and melanomas ${ }^{36}$. During 5-ALA based PDT, absorption of photon energy by the photosensitizer (PpIX) contained in tumor tissue induces an oxygen dependent photochemical reaction that most probably results in the generation of toxic reactive singlet oxygen species (ROSs) ${ }^{42}$. The oxidative damageoccurs in close proximity to the site where ROSs formed. Since we have demonstrated that eEF1A1 binds to photosensitizer PpIX, ROS generated during PDT will deactive eEF1A1 and eliminate its functions, which may lead to cell death. Moreover, eEF1A1 protein shares $75 \%$ nucleotide and $96 \%$ amino acid homology with its isoform eEF1A2 protein, which is also reported as diagnostic marker for cancers ${ }^{26,43}$. This suggests eEF1A2 as another potential target for 5-ALA mediated PDT.

In conclusion, this study shows the intracellular interaction between ALA-induced PpIX and eEF1A1. Furthermore, the accumulation of PpIX is correlated with eEF1A1 expression, which has provided new insights in both the mechanism of PpIX accumulation in cancer cells in 5-ALA based PDT as well as how the PDT works (target). Our study has suggested eEF1A1 expresson as a diagnosis parameter for determining therapeutic strategies, since 5-ALA based PDT might be specifically effective in treating patients who have high-expression of eEF1A1. 


\begin{abstract}
Methods
Phage displayed cDNA library screen. Phage displayed cDNA library technology is a well-designed and high-throughput protein screening method based on expressing recombinant random peptides on the surface of filamentous phage and screening functional peptides, such as RNA-binding proteins ${ }^{22}$, drug targeted proteins ${ }^{23}$, protein targeting peptides ${ }^{24}$ and cell targeting peptides ${ }^{25}$. To screen the PPIX-affinitive protein, we used the high-throughput T7 select human liver cDNA Library (Merck, Darmstadt, Germany) screening. In this library, peptides transcribed from human liver cDNA were exposed in the surface of T7 phages. To keep a high vitality, the library was amplified in host bacteria BLT5403 (Merck) to reach a titer of $\sim 10^{10} \mathrm{PFU} / \mathrm{ml}$ for the screening.

In screening, the 96-well plate was coated with PpIX $(1 \mu \mathrm{g} / \mathrm{ml}, 200 \mu \mathrm{l} /$ well, Frontier Scientific, Logan, UT, USA) in darkness at $4^{\circ} \mathrm{C}$ overnight. The 1:100 diluted amplified phages $(200 \mu \mathrm{l} /$ well $)$ were introduced into the plate and incubated at $37^{\circ} \mathrm{C}$ for $30 \mathrm{~min}$ with gentle rocking. After five washes with phosphate buffered saline (PBS), BLT5403 were added into the wells to harvest the target phages. The eluted BLT5403 containing target phages were amplified and subjected to additional rounds of selection. The titers of phages in each round were monitored to assess the enrichment of phages during screening. Four rounds of the affinity selection process were performed (Fig. 1a) to screen out PpIX binding proteins.
\end{abstract}

Cloning of the phages presenting PpIX binding proteins. The screened out phages were plated at low density (less than $100 \mathrm{PFU} /$ plate). The plaques were transferred onto nitrocellulose (NC) filter membrane (Schleicher \&Schuell, Dassel, Germany) in situ at $37^{\circ} \mathrm{C}$ for $1 \mathrm{~h}$. After three washes with PBS, NC membrane was incubated in $1 \mathrm{ug} / \mathrm{ml}$ PpIX with gentle shaking at $37^{\circ} \mathrm{C}$ for $30 \mathrm{~min}$ and imaged by epifluorescence microscope (Olympus IX51, Olympus Corp., Tokyo, Japan, Ex: $530 \mathrm{~nm} / \mathrm{Em}: 630 \mathrm{~nm}$ ). The plaque with highest PpIX fluorescence was cloned from the plate.

To verify the PpIX binding of selected phage clone, lysate smear (including lysogenic bacteria and phage) of selected phage clone and control (no PpIX binding) were incubated with or without $1 \mu \mathrm{g} / \mathrm{ml} \mathrm{PpIX}$ at $37^{\circ} \mathrm{C}$ for $30 \mathrm{~min}$ and imaged by fluorescence microscope. In some experiments, rabbit anti-human eEF1A1 polyclonal antibody ( $10 \mathrm{ug} / \mathrm{ml}, 37^{\circ} \mathrm{C}, 10 \mathrm{~min}$, Santa Cruz Biotech Inc., Santa Cruz, CA, USA) was used to block PpIX binding of selected phage clone. The mean florescence intensity (MFI) of phage clones in fluorescence images were analyzed by "analyze particles" in FIJI-Image $2^{44}$.

Identification of protein presented by selected phage clone. Encoding sequences of PpIX binding peptide in selected phage clone were amplified by PCR with the phage lysate as the template. The primers used in PCR were designed as T7 UP Primer $5^{\prime}$-AACCCCTCAAGACCCGTTTA- $3^{\prime}$ and T7 DOWN primer $5^{\prime}$-GGAGCTGTCGTATTCCAGTC-3'. The PCR products were analyzed by agarose electrophoresis. The inserted gene was sequenced by Invitrogen (Shanghai, China). Homology analysis between obtained sequences and human genes listed in GeneBank (National Center for Biotechnology Information) were performed by using BLAST.

Plasmid construction. cDNA of human eEF1A1 gene were amplified from total mRNA of human fetal liver (BiocolorBioScience\& Technology, Shanghai, China) by reverse transcription-polymerase chain reaction (RT-PCR). The primers used in PCR were designed as $5^{\prime}$-CTGAATTCATGGGAAAGGAAAAGACT- $3^{\prime}$ and $5^{\prime}$-ACTGGATCCTTTAGCCTTCTGAGCTTTCTG-3'. The eEF1A1 cDNA were cloned into pEGFP-C1 (BD Clontech, Palo Alto, CA) and verified by PCR, restriction enzyme digestion and DNA sequencing (Invitrogen).

Cell culture and transfection. HepG2 cells and L02 cells were purchased from Cell Bank of Shanghai Institute of Biochemistry and Cell Biology (Chinese Academy of Sciences, Shanghai, China). Cells were used in related experiments within 6 month after received the cells from the bank. The cells were performed short tandem repeat (STR) and mycoplasma tests in the bank. Cells were cultured in RPMI 1640 complete medium (Gibco, Rockville, MD) containing 10\% fetal bovine serum (HyClone, South Logan, UT) at $37^{\circ} \mathrm{C}$ with $5 \% \mathrm{CO}_{2}$. For transfection, empty (pEGFP-C1) or eEF1A1-reconstructed plasmids were transfected into HepG2 cells using Lipofectamine 2000 (Life Technologies, Shanghai, China) according to the manufacturer's instructions.

qRT-PCR. RNA in L02 cells, HepG2 cells and transfected HepG2 cells was extracted using TRIzol (Invitrogen). cDNA was synthesized using FastQuant RT Kit (with gDNase) (TIANGEN, Beijing, China) according to the manufacturer's instructions. Expression level of eEF1A1 mRNA was measured by Roche Lightcycler 480 (Roche, Nutley, NJ, USA) using SuperRealPreMix Plus (SYBR Green, TIANGEN) and normalized to expression level of GAPDH.

Western blot. Cell lysates of L02 cells, HepG2 cells and transfected HepG2 cells were fractionated by sodium dodecyl sulfate polyacrylamide gel electrophoresis (SDS-PAGE) and detected by immunoblotting using rabbit anti-human eEF1A1 polyclonal antibody (1:1000) and horseradish peroxidase (HRP)-conjugated goat anti-rabbit antibody (1:5000, Santa Cruz Biotech Inc.). Sample loading amounts in the assay were normalized by GAPDH (rabbit anti-human GAPDH polyclonal antibody, 1:2000, Santa Cruz Biotech Inc.) blotting. The blots were visualized by G:BOX Gel \& Blot Imaging Systems (Syngene, Cambridge, England).

Fluorescence binding assay. The 96-well plate was coated by recombinant human eEF1A1 (Abnova, Taipei, Taiwan) or control BSA (Sigma, Fremont, CA, USA) with a series of concentrations $(200-1000 \mathrm{ng} / 100 \mu \mathrm{l})$ at $4^{\circ} \mathrm{C}$ overnight. After three washes with PBS, PpIX $(1 \mu \mathrm{g} / \mathrm{ml}, 200 \mu \mathrm{l} /$ well $)$ were added into the plate and incubated at $37^{\circ} \mathrm{C}$ for $1.5 \mathrm{~h}$. After three washes with PBS, the fluorescence of PpIX was measured in SpectraMax M5 microplate reader (Molecular Devices Inc., Sunnyvale, CA, USA). The excitation and emission wavelength during the measurements are 400 and $620 \mathrm{~nm}$, respectively. 
SPR assay. Label-free SPR based technology ${ }^{45}$ for studying biomolecular interactions in real time was used to assess the $\mathrm{K}_{\mathrm{a}}$ of eEF1A1-PpIX interaction. SPR measurements were conducted following manufacturer's protocol. Briefly, the eEF1A1 as ligand was immobilized to a CM5 sensory chip via amine coupling. A reference chamber was processed by ethanolamine. Various concentrations of PpIX were injected into the chip. The response curves, calculated as the difference between responses in the sample chamber and the reference chamber, were recorded to assess the binding. The sensor chip was regenerated with 2 molar $\mathrm{NaCl}$ between measurements. The sensorgrams were fitted using a one-site binding model.

Immunofluorescence. To monitor the intracellular localizations of eEF1A1 and ALA-induced PpIX, HepG2 cells were cultured with medium containing 5-ALA ( $1 \mathrm{mM}$, Sigma) for 24 hours and fixed by $4 \%$ paraformaldehyde for $10 \mathrm{~min}$ at room temperature (RT). The cells were permeabilized by $0.5 \%$ Triton X-100 (Sigma) for $1 \mathrm{~h}$ at RT and treated with blocking buffer containing 2\% BSA, 10\% goat serum (Gibco) and 0.1\% Tween-200 (Sigma) for $1 \mathrm{~h}$. The samples were incubated with rabbit anti-human eEF1A1 polyclonal antibody $(10 \mu \mathrm{g} / \mathrm{ml})$ at $4{ }^{\circ} \mathrm{C}$ overnight and incubated with fluorescein isothiocyanate (FITC)-conjugated goat anti-rabbit antibody $(10 \mu \mathrm{g} /$ $\mathrm{ml}$, Boster Biotechnology Corp., Wuhan, China) at $37^{\circ} \mathrm{C}$ for $30 \mathrm{~min}$. All the operations were protected from light.

Microscopy. Fluorescence image of eEF1A1 and ALA-induced PpIX in immunofluorescence samples were acquired by a CLSM (Leica TCS SP5, Leica Microsystems CMS GmbH, Am Friendenplatz, Mannheim, Germany) using a $40 \times$ oil-immersion objective $(\mathrm{NA}=1.25)$ as described before ${ }^{46-49}$. FITC-labeled eEF1A1 was excited by $488 \mathrm{~nm}$ argon laser and detected by a photomultiplier tube (PMT) with the bandpass filter of 500-550 nm. ALA-induced PpIX was co-excited by $405 \mathrm{~nm}$ and $561 \mathrm{~nm}$ lasers (around the two peaks of PpIX excitation) to achieve maximum excitation and detected by a PMT with the bandpass filter of 590-650 nm.

Intracellular localizations of eEF1A1 and ALA-induced PpIX were also assessed by super resolution SIM imaging (N-SIM microscopy system on a Ti microscope, Nikon Instruments Inc, Melville, NY) with a resolution of $\sim 100 \mathrm{~nm}^{29}$. The samples were imaged with a 100X oil-immersion objective (NA=1.49) and an Andor DU-897 EM-CCD camera (Andor Technology PLC, Belfast, Northern Ireland). A $488 \mathrm{~nm}$ and a $561 \mathrm{~nm}$ laser beam were used to excite FITC-labeled eEF1A1 and PpIX, respectively.

Intensity correlation analysis. The intensity correlation analysis was done by evaluating Pearson's correlation coefficient $(\mathrm{Rr}$, range $-0.12-1)$, Manders' overlap coefficient $(\mathrm{R}$, range $0-1)$ and co-localization coefficient (M1, M2, range $0-1)^{30}$ and performing intensity correlation analysis (ICA) ${ }^{31}$ with Image plugin as described previously ${ }^{30,31,50}$. For R, Rr, M1 and M2, a value of 1 represents total colocalization while a value of 0 represents no colocalization. For ICA, the output - intensity correlation quotient (ICQ) is from the range of -0.5-0.5. A value from $0-0.5$ indicates the intensities of the two channels vary synchronically.

Accumulation of ALA-induced PpIX. The HepG2 cells and L02 cells (8,000 cells/well) were seeded in 96-well plates and cultured for 6 hours prior to assays. During the assay, cells were incubated in serum-free RPMI1640 medium with or without 1 mM 5-ALA in darkness. Time-lapse measurements of PpIX fluorescence were performed by SpectraMax M5 microplate reader as described above. Soluble 5-ALA or PpIX in medium were washed out before measurements so that the fluorescence only represents intercellular PpIX. In some experiments, HepG2 cells transfected with empty or eEF1A1 reconstructed plasmid were served as experimental or control groups.

PpIX degradation assay. HepG2 cells were lysed by nondenaturing lysis buffer (Cell Signaling Technology Inc, Danvers, MA, USA) containing protease inhibitor PMSF (phenylmethylsulfonyl fluoride, $1 \mathrm{mM}$, Life Technologies) on ice for $10 \mathrm{~min}$. The cell lysate was mixed with PpIX $(1 \mu \mathrm{g} / \mathrm{ml})$ and eEF1A1 $(1.3 \mu \mathrm{g} / \mathrm{ml}, \mathrm{Abnova})$. The lysate mixed with PpIX was used as a control group. The lysate mixed with vehicles or eEF1A1 were used as blank controls for normalization. Time-lapse measurements of PpIX fluorescence were performed by SpectraMax M5 microplate reader as described above. The lysate were incubated at $37^{\circ} \mathrm{C}$ between the measurements.

Statistics. Statistical analysis was performed with Prism 6 (GraphPad). Data were presented as mean \pm standard deviation (SD). Single data points were presented in some graphs. The means for the data sets were compared, each separately, using student t-tests with equal variances. Linear regressions were performed for some data sets. The slopes of the linear regressions for the data sets were compared using an F-test. P values less than 0.05 were considered significant.

\section{References}

1. Ferrario, A. et al. The matrix metalloproteinase inhibitor prinomastat enhances photodynamic therapy responsiveness in a mouse tumor model. Cancer Res. 64, 2328-2332 (2004).

2. Sur, B. W., Nguyen, P., Sun, C. H., Tromberg, B. J. \& Nelson, E. L. Immunophototherapy using PDT combined with rapid intratumoral dendritic cell injection. Photochem. Photobiol. 84, 1257-1264 (2008).

3. Mallidi, S., Spring, B. Q., Chang, S., Vakoc, B. \& Hasan, T. Optical imaging, photodynamic therapy and optically triggered combination treatments. Cancer J. 21, 194-205 (2015).

4. Spring, B. Q., Rizvi, I., Xu, N. \& Hasan, T. The role of photodynamic therapy in overcoming cancer drug resistance. Photochem. Photobiol. Sci. 14, 1476-1491 (2015)

5. Chen, W. \& Zhang, J. Using nanoparticles to enable simultaneous radiation and photodynamic therapies for cancer treatment. J. Nanosci. Nanotechnol. 6, 1159-1166 (2006).

6. Hunter, G. A. \& Ferreira, G. C. Molecular enzymology of 5-aminolevulinate synthase, the gatekeeper of heme biosynthesis. Biochim. Biophys. Acta 1814, 1467-1473 (2011).

7. Solban, N., Rizvi, I. \& Hasan, T. Targeted photodynamic therapy. Lasers Surg. Med. 38, 522-531 (2006).

8. Hua, Z., Gibson, S. L., Foster, T. H. \& Hilf, R. Effectiveness of delta-aminolevulinic acid-induced protoporphyrin as a photosensitizer for photodynamic therapy in vivo. Cancer Res. 55, 1723-1731 (1995). 
9. Chiu, L. L. et al. Photodynamic therapy on keloid fibroblasts in tissue-engineered keratinocyte-fibroblast co-culture. Lasers Surg. Med. 37, 231-244 (2005).

10. Ji, J. et al. Improvement of DC vaccine with ALA-PDT induced immunogenic apoptotic cells for skin squamous cell carcinoma. Oncotarget 6, 17135-17146 (2015)

11. Keefe, K. A. et al. Photodynamic therapy of high-grade cervical intraepithelial neoplasia with 5-aminolevulinic acid. Lasers Surg. Med. 31, 289-293 (2002).

12. Hagiya, Y. et al. Expression levels of PEPT1 and ABCG2 play key roles in 5-aminolevulinic acid (ALA)-induced tumor-specific protoporphyrin IX (PpIX) accumulation in bladder cancer. Photodiagnosis Photodyn. Ther. 10, 288-295 (2013).

13. Ishikawa, T., Kajimoto, Y., Inoue, Y., Ikegami, Y. \& Kuroiwa, T. Critical role of ABCG2 in ALA-photodynamic diagnosis and therapy of human brain tumor. Adv. Cancer Res. 125, 197-216 (2015).

14. Hirschberg, H. et al. Repetitive photodynamic therapy of malignant brain tumors. J. Environ. Pathol. Toxicol. Oncol. 25, 261-279 (2006).

15. Takahashi, K. et al. Antitumor effect of combination of hyperthermotherapy and 5-aminolevulinic acid (ALA). Anticancer Res. 33, 2861-2866 (2013).

16. Hino, H. et al. 5-Aminolevulinic acid-mediated photodynamic therapy using light-emitting diodes of different wavelengths in a mouse model of peritoneally disseminated gastric cancer. J. Surg. Res. 185, 119-126 (2013).

17. Yu, C. H. \& Yu, C. C. Photodynamic therapy with 5 -aminolevulinic acid (ALA) impairs tumor initiating and chemo-resistance property in head and neck cancer-derived cancer stem cells. PLoS One 9, e87129 (2014).

18. Zawacka-Pankau, J. et al. Protoporphyrin IX interacts with wild-type p53 protein in vitro and induces cell death of human colon cancer cells in a p53-dependent and -independent manner. J. Biol. Chem. 282, 2466-2472 (2007).

19. Furre, I. E. et al. Targeting PBR by hexaminolevulinate-mediated photodynamic therapy induces apoptosis through translocation of apoptosis-inducing factor in human leukemia cells. Cancer Res. 65, 11051-11060 (2005).

20. Zawacka-Pankau, J. et al. Tumor suppressor Fhit protein interacts with protoporphyrin IX in vitro and enhances the response of HeLa cells to photodynamic therapy. J. Photochem. Photobiol. B. 86, 35-42 (2007).

21. Silva, J. N. et al. Photodynamic therapies: principles and present medical applications. Biomed. Mater. Eng. 16, S147-154 (2006).

22. Danner, S. \& Belasco, J. G. T7 phage display: a novel genetic selection system for cloning RNA-binding proteins from cDNA libraries. Proc. Natl. Acad. Sci. USA 98, 12954-12959 (2001).

23. Schaffer, L. et al. Assembly of high-affinity insulin receptor agonists and antagonists from peptide building blocks. Proc. Natl. Acad. Sci. USA 100, 4435-4439 (2003).

24. Levenhagen, M. A. et al. Structural and functional characterization of a novel scFv anti-HSP60 of Strongyloides sp. Sci. Rep. 5, 10447 (2015).

25. Nowakowski, G. S. et al. A specific heptapeptide from a phage display peptide library homes to bone marrow and binds to primitive hematopoietic stem cells. Stem Cells 22, 1030-1038 (2004).

26. Abbas, W., Kumar, A. \& Herbein, G. The eEF1A Proteins: At the Crossroads of Oncogenesis, Apoptosis, and Viral Infections. Front. Oncol. 5, 75 (2015).

27. Brancaleon, L. \& Moseley, H. Effects of photoproducts on the binding properties of protoporphyrin IX to proteins. Biophys. Chem. 96, 77-87 (2002).

28. Mouquet, H. et al. Polyreactivity increases the apparent affinity of anti-HIV antibodies by heteroligation. Nature 467, 591-595 (2010).

29. Komis, G. et al. Superresolution live imaging of plant cells using structured illumination microscopy. Nat. Protoc. 10, 1248-1263 (2015).

30. Manders, E. M. M., Verbeek, F. J. \& Aten, J. A. Measurement of co-localization of objects in dual-colour confocal images. J. Microsc 169, 375-382 (1993).

31. Li, Q. et al. A syntaxin 1, Galpha(o), and N-type calcium channel complex at a presynaptic nerve terminal: analysis by quantitative immunocolocalization. J. Neurosci. 24, 4070-4081 (2004).

32. Grassi, G. et al. The expression levels of the translational factors eEF1A $1 / 2$ correlate with cell growth but not apoptosis in hepatocellular carcinoma cell lines with different differentiation grade. Biochimie 89, 1544-1552 (2007).

33. Byun, H. O. et al. Cathepsin D and eukaryotic translation elongation factor 1 as promising markers of cellular senescence. Cancer Res. 69, 4638-4647 (2009).

34. Browne, G. J. \& Proud, C. G. Regulation of peptide-chain elongation in mammalian cells. Eur. J. Biochem. 269, 5360-5368 (2002).

35. Gross, S. R. \& Kinzy, T. G. Translation elongation factor $1 \mathrm{~A}$ is essential for regulation of the actin cytoskeleton and cell morphology. Nat. Struct. Mol. Biol. 12, 772-778 (2005).

36. Blanch, A., Robinson, F., Watson, I. R., Cheng, L. S. \& Irwin, M. S. Eukaryotic translation elongation factor 1-alpha 1 inhibits p53 and p73 dependent apoptosis and chemotherapy sensitivity. PLoS One 8, e66436 (2013).

37. Vera, M. et al. The translation elongation factor eEF1A1 couples transcription to translation during heat shock response. Elife 3, e03164 (2014).

38. Itagaki, K. et al. Eukaryotic translation elongation factor 1A induces anoikis by triggering cell detachment. J. Biol. Chem. 287, 16037-16046 (2012).

39. Chuang, S. M. et al. Proteasome-mediated degradation of cotranslationally damaged proteins involves translation elongation factor 1A. Mol. Cell. Biol. 25, 403-413 (2005).

40. Lin, K. W., Yakymovych, I., Jia, M., Yakymovych, M. \& Souchelnytskyi, S. Phosphorylation of eEF1A1 at Ser300 by TbetaR-I results in inhibition of mRNA translation. Curr. Biol. 20, 1615-1625 (2010).

41. Krastel, P. et al. Nannocystin A: an Elongation Factor 1 Inhibitor from Myxobacteria with Differential Anti-Cancer Properties. Angew. Chem. Int. Ed. Engl. 54, 10149-10154 (2015).

42. Jiang, X. et al. Toluidine blue $\mathrm{O}$ and porphyrin-mediated photodynamic therapy on three main pathogenic bacteria of periodontitis using portable LED phototherapy device. J. Innov. Opt. Heal. Sci. 08, 1550017 (2015).

43. Tomlinson, V. A. et al. Expression of eEF1A2 is associated with clear cell histology in ovarian carcinomas: overexpression of the gene is not dependent on modifications at the EEF1A2 locus. Br. J. Cancer 96, 1613-1620 (2007).

44. Schindelin, J. et al. Fiji: an open-source platform for biological-image analysis. Nat. Methods 9, 676-682 (2012).

45. Yan, C. et al. Discovery and characterization of small molecules that target the GTPase Ral. Nature 515, 443-447 (2014).

46. Fan, Z. C. et al. Real-time monitoring of rare circulating hepatocellular carcinoma cells in an orthotopic model by in vivo flow cytometry assesses resection on metastasis. Cancer Res. 72, 2683-2691 (2012).

47. Lin, W. et al. The bullseye synapse formed between $\mathrm{CD} 4^{+} \mathrm{T}$-cell and staphylococcal enterotoxin B-pulsed dendritic cell is a suppressive synapse in T-cell response. Immunol. Cell. Biol. 93, 99-110 (2015).

48. Lin, W. et al. Morphological change of $\mathrm{CD}^{+} \mathrm{T}$ cell during contact with $\mathrm{DC}$ modulates $\mathrm{T}$-cell activation by accumulation of $\mathrm{F}$-actin in the immunology synapse. BMC Immunol. 16, 49 (2015).

49. Guo, J., Fan, Z., Gu, Z. \& Wei, X. Studying the role of macrophages in circulating prostate cancer cells by in vivo flow cytometry. J. Innov. Opt. Heal. Sci. 05, 1250027 (2012)

50. Mravec, J. et al. Subcellular homeostasis of phytohormone auxin is mediated by the ER-localized PIN5 transporter. Nature 459, $1136-1140$ (2009). 


\section{Acknowledgements}

This work was supported by the grants of the National Major Scientific Research Program of China (Grant No. 2011CB910404 and 2012CB966801), the National Nature Science Foundation of China (Grant No. 61227017 and 81272818) and the National Science Fund for Distinguished Young Scholars (Grant No. 61425006) and the Fudan University 2010 Excellent Ph.D. Research Foundation.

\section{Author Contributions}

Experiments were designed by Z.F. and X.W. Experiments were performed by X.C., Z.F. and W.L. Data analysis was performed by Z.F. Image processing was performed by Z.F. and D.W. The manuscript was written by Z.F., X.C., D.W., W.L., B.L. and X.W. The project was supervised by X.W. and N.Z. All authors discussed the results and commented on the manuscript.

\section{Additional Information}

Competing financial interests: The authors declare no competing financial interests.

How to cite this article: Fan, Z. et al. eEF1A1 binds and enriches protoporphyrin IX in cancer cells in 5-aminolevulinic acid based photodynamic therapy. Sci. Rep. 6, 25353; doi: 10.1038/srep25353 (2016).

(c) (i) This work is licensed under a Creative Commons Attribution 4.0 International License. The images or other third party material in this article are included in the article's Creative Commons license, unless indicated otherwise in the credit line; if the material is not included under the Creative Commons license, users will need to obtain permission from the license holder to reproduce the material. To view a copy of this license, visit http://creativecommons.org/licenses/by/4.0/ 